\title{
STONE-WEIERSTRASS THEOREMS FOR $C(X)$ WITH THE SEQUENTIAL TOPOLOGY
}

\author{
ZDENĚK FROLIK
}

\begin{abstract}
The spaces $P$ with one of the following two properties are studied: every continuous function is a Baire function with respect to any algebra $Q$ of continuous functions such that $Q$ projectively generates the topology, or with respect to any algebra which distinguishes the points. The former property is equivalent to the statement that of any pair of disjoint zero sets at least one is Lindelöf, the latter implies that the space is Lindelöf and is implied by analyticity. Connections with the Blackwell problem are shown.
\end{abstract}

The main results are Theorems 2 and 3 below. Note that by a function we always mean a real valued function, and by an algebra of functions we mean an algebra of functions (with the usual "pointwise" operations) which contains all constant functions. Unless explicitly stated otherwise, by a space we mean a completely regular topological space. In general we use the terminology and notation of [1]. In $\S 1$, the results will be stated, and the proofs of results called theorems will be given in $\S 2$.

1. The collection Baire $(P)$ of all Baire sets in a topological space $P$ is the smallest $\sigma$-algebra of subsets of $P$ such that all continuous functions are measurable. Recall that $\operatorname{Baire}(P)$ is the smallest countably additive and countably multiplicative collection of sets, which contains all zero sets (equivalently, cozero sets) in $P$. $A$ space $P$ locally belongs to a collection $\mathrm{N} /$ of subsets of $P$ if each point of $P$ has arbitrarily small neighborhoods in $\mathfrak{T}$. We are prepared to state our first result.

TheOREM 1. A space $P$ is Lindelö if and only if the collection of all Baire sets in $P$ is the smallest countably additive and countably multiplicative collection $\mathfrak{T H}$ of sets such that $P$ locally belongs to $\mathfrak{T}$.

In order to state the next result we need more notation and terminology. Denote by $F(P)$ the algebra of all functions on $P$. For each $M \subset F(P)$ let $u M$ be the set of all pointwise limits of sequences in $M$.

Received by the editors March 21, 1970.

AMS 1969 subject classifications. Primary 2810, 5440, 2635; Secondary 4625.

Key words and phrases. Lindelöf, almost Lindelöf, Baire-minimal, Blackwell space, analytic, algebra of continuous functions, Baire set, Baire function, sequential topology. 
It may happen that $u u M \neq u M$, but in any case we let $s M$ denote the smallest set $X \supset M$ for which $u X=X$. The relation $\{M \rightarrow s M\}$ is a topological closure operation, and the corresponding topology is called the pointwise sequential topology on $F(P)$. For each countable ordinal $\alpha$ define

$$
u_{\alpha} M=u \cup\left\{u_{\beta} M \mid \beta<\alpha\right\}
$$

where $u_{0} M=M$. Clearly $s M=\bigcup\left\{u_{\alpha} M \mid \alpha<\omega_{1}\right\}$. The elements of $s M$ are called the Baire functions with respect to $M$; the elements of $u_{\alpha} M$ are the Baire functions of class $\alpha$; and the smallest $\alpha$ with $f \in u_{\alpha} M$ is called the order of $f$ with respect to $M$. By the pointwise sequential topology on a subset of $F(P)$ we mean the relativization of the sequential topology in $F(P) .^{1}$

We say that a family $\left\{f_{a}\right\}$ of functions on a space $P$ projectively generates the topology of $P$ if the topology of $P$ is the coarsest topology such that each $f_{a}$ is continuous.

THEOREM 2. The following conditions $\mathrm{A}-\mathrm{F}$ on a space $P$ are equivalent:

A. Of any pair of disjoint zero sets in $P$, at least one is Lindelöf.

B. Baire $(P)$ is the smallest $\sigma$-algebra $\mathfrak{T C}$ of subsets of $P$ such that $P$ locally belongs to $\mathrm{Tr}$.

C. Every embedding of $P$ is a Baire embedding. That is, if $P \subset Q$ then each Baire set in $P$ is a trace on $P$ of a Baire set in $Q$.

$D$. If an algebra $Q$ of bounded continuous functions on $P$ projectively generates $P$, then $a$ is dense in $C(P)$ in the pointwise sequential topology.

E. With the assumption in $\mathrm{D}, u Q \supset C(P)$.

$\mathrm{F}$. With the assumption in $\mathrm{D}$, if $\mathrm{Q}$ is a lattice then every continuous function $f$ on $P$ which is bounded from below is a pointwise limit of an increasing sequence $\left\{f_{n}\right\}$ in a (and hence, $\left\{f_{n}\right\}$ converges to $f$ in the compact-open topology by Dini's theorem).

Definition 1. A space $P$ will be called almost Lindelöf if the conditions in Theorem 2 are fulfilled.

It follows immediately from condition A that every paracompact almost Lindelöf space is Lindelöf. If, in condition A, we replace "Lindelöf" by "compact" we obtain Hewitt's "almost compact spaces" [7]. (It should be remarked that Hewitt's "almost compact spaces" are often called the "spaces with unique uniform structure," and the term "almost compact" is used for " $H$-closed spaces.") Clearly

1 The sequential pointwise topology on $X \subset F(P)$ need not be sequential. For example let $X=M \cup(f)$ where $f \in u_{2} M-u_{1} M$. See remark in 2.3 for an example related to Theorem 3. 
every almost compact space is almost Lindelöf. On the other hand, an almost Lindelöf space need not be Lindelöf nor almost compact; for example, remove one cluster point from the space of countable ordinals. Recall that [6, Theorem 3] every embedding of $P$ is a $Z$ embedding if and only if $P$ is either Lindelöf or almost compact. Finally observe that condition $\mathrm{A}$ is equivalent to the statement that $v P$ is Lindelöf and $v P-P$ is void or a singleton $[6,4.3]$. It follows that a space $P$ is Lindelöf if and only if it is almost Lindelöf and realcompact. (Compare this result with E. Hewitt's theorem [7] characterizing the almost compact spaces as those spaces $P$ for which $\beta P-P$ is empty or a singleton.)

THEOREM 3. If $P$ is an analytic space then the following statement is true:

If an algebra $Q$ of continuous functions on $P$ distinguishes the points of $P$ then $a$ is dense in $C(P)$ in the sequential topology.

Definition 2. A space $P$ is called Baire-minimal if $P$ enjoys the property of analytic spaces in Theorem 3.

By Theorem 3 every analytic space is Baire-minimal. The following example shows that a Baire-minimal space need not be analytic.

ExAmple. For any uncountable set $P$, and point $x$ in $P$, define a topology on $P$ as follows: a set $U \subset P$ is open if and only if either $x \notin U$ or $x \in U$ and $P-U$ is countable. Then $X \subset P$ is a Baire set in $P$ if and only if either $x \notin X$ and $X$ is countable, or $x \in X$ and $P-X$ is countable. It is easy to show that $P$ is Baire-minimal, and we shall prove that $P$ is not analytic. Recall that a space $Q$ is called analytic [3], [4] if there exists an usco-compact (that is, upper semicontinuous compact-valued) correspondence of the space of irrational numbers onto $Q$. We shall prove more; namely that $P$ is not the usco-compact image of any space with a countable base for open sets. Assume that $f$ is an usco-compact correspondence of a second countable space $T$ onto $P$; we shall derive a contradiction. First we show that each $t$ in $T$ has a neighborhood $U_{t}$ such that $f\left[U_{t}\right] \subset f[(t)]$. Given any point $t$ in $T$, let $\left\{V_{n}\right\}$ be a local base $\left\{V_{n}\right\}$ at $t$. If $f\left[V_{n}\right] \subset f[(t)]$ for no $n$, then there would exist a countable set $X \subset P-f[(t)]$ which would meet each $f\left[V_{n}\right]$, and this would contradict the upper semicontinuity of $f$ at $t$ since $P-X$ is a neighborhood of $f\left[V_{n}\right]$. Next, since $T$ is Lindelöf, $\left\{U_{t}\right\}$ has a countable subcover, and hence there exists a countable $Y \subset T$ with $f[Y]=P$. Finally, each compact subset of $P$ is finite, and hence $f[Y]$ is countable, and this contradicts our assumption that $P$ is uncountable.

Let $K$ be the set $P$ in the above example with the compact topology 
such that $x$ is the only cluster point of $K$. Evidently

$$
\operatorname{Baire}(P)=\operatorname{Baire}(K) \text {. }
$$

I do not know of an example of a Baire-minimal space which is not Baire equivalent with an analytic space.

Obviously every Baire-minimal space is almost Lindelöf.

\section{Theorem 4. Every Baire-minimal space is Lindelöf.}

On the other hand a Lindelöf metrizable space need not be Baireminimal. For example, take a set $X$ in a separable metrizable space $P$ such that $X$ is not a Baire set in $P$. Denote by $Q$ the set $P$ endowed with a topology such that $X$ and $P-X$ are subspaces of both $P$ and $Q$, and $X$ is both open and closed in $Q$. The identity mapping of $Q$ onto $P$ is continuous, but $\operatorname{Baire}(P) \neq \operatorname{Baire}(Q)$.

Definition 3. A measurable space is called a Blackwell space if the $\sigma$-algebra of measurable sets is the only countably generated $\sigma$ algebra of measurable sets which distinguishes points. A topological space is called a Blackwell space if $P$ is a separable metrizable space such that $\langle P, \operatorname{Baire}(P)\rangle$ is a Blackwell measurable space.

It should be remarked that every analytic metrizable space is a Blackwell space, and it is not known if the converse is true. If we assume the continuum hypothesis then every metrizable topologization of a Blackwell space is separable. Indeed, then the $\sigma$-algebra of all Baire sets of no nonseparable space is countably generated.

Theorem 5. Every Blackwell space is Baire-minimal. A countably generated measurable space $P$ is a Blackwell space if and only if each separable metrizable topologization of $P$ is a Blackwell topological space.

2. Proofs. We start with four very simple lemmas which will be applied several times.

Lemma 1. Assume that a space $P$ locally belongs to countably additive and countably multiplicative collection of sets $\mathcal{C}$. If $P$ is not Lindelöf then there exists a countably additive and countably multiplicative collection $\mathfrak{M C} \subset \mathrm{C}$ such that $P$ locally belongs to $\mathfrak{M}$, and if $X \in \mathfrak{M}$, then $P-X$ $\notin \Re$.

Proof. If $u$ is an open cover of $P$ which contains no countable subcover then the set $\mathfrak{T}$ of all sets $C$ in $\mathcal{C}$ which are covered by a countable subcollection of $\mathcal{U}$ has the properties in Lemma 1.

Lemma 2. Assume that a space $P$ locally belongs to a collection of sets TT. If $U$ is a neighborhood of a Lindelöf subspace $X$ of $P$ then $X \subset M$ $\subset U$ for some union $M$ of a countable family in $\mathfrak{T}$. 
Proof. Obvious.

Lemma 3. Let $M \subset F(P)$, and let $\mathfrak{T}$ be the smallest $\sigma$-algebra of subsets of $P$ such that every $f:\langle P, \mathfrak{T}\rangle \rightarrow R, f \in M$, is measurable. Then every element of $s M$ is measurable. Furthermore, if $X \in \mathbb{N}$ then there exists a countable subset $M^{\prime} \subset M$ such that if $x \in X$ and $y \notin X$ then fx $\neq$ fy for some $f$ in $M^{\prime}$. (We shall say that $M^{\prime}$ distinguishes $X$.)

Proof. Every element of $s M$ is measurable because the pointwise limit of any sequence of measurable functions is measurable. To prove the second statement observe that the collection of all "distinguishable" sets is a $\sigma$-algebra.

Lemma 4. Condition A in Theorem 2 is satisfied if and only if for each continuous function $f$ on $P$ there exists a real number $r$ such that the set $E\{x \mid f x \neq r\}$ is Lindelöf.

ProOF. "If" is obvious, and to prove "only if" consider the set $S$ of all real numbers $s$ such that $E\{x \mid f x<s\}$ is Lindelöf. Observe that $P$ is Lindelöf if $S$ has no upper bound, and that if $S$ has an upper bound, then the supremum $r$ of $S$ has the property stated in Lemma 4.

2.1. Proof of Theorem 1. If $P$ is not Lindelöf then $\mathfrak{T}$ in Lemma 1 does not contain Baire $(P)$. Assume that $P$ is Lindelöf, and $\mathscr{T}$ is a countably additive and countably multiplicative collection of sets such that $P$ locally belongs to $\mathscr{T}$. It is enough to prove that every zero set $Z$ belongs to $\Re$. Since $Z$ is a $G_{\delta}$, by Lemma 2 the set $Z$ is a countable intersection of elements of $\mathfrak{T}$ and hence belongs to $\mathfrak{T}$.

2.2. Proof of Theorem 2.

2.2.1. First we shall show that each of the conditions $B-F$ in Theorem 2 implies condition A. Assume that condition A is not fulfilled. Then there exist two disjoint zero sets $Z_{1}$ and $Z_{2}$ such that neither $Z_{1}$ nor $Z_{2}$ is Lindelö. Take a countably additive open cover $\mathcal{u}$ of $P$ such that neither $Z_{1}$ nor $Z_{2}$ is contained in an element of $\mathcal{u}$; thus $\mathcal{U}$ is uncountable. Let $a$ be the set of all continuous functions $f$ on $P$ such that $f$ is constant on the complement of an element of $\mathcal{u}$, and let $Q^{*}$ be the set of all bounded functions in $a$. Clearly $Q$ and $Q^{*}$ are uniformly closed algebras which projectively generate the topo$\operatorname{logy}$ of $P$. Let $K$ be a compactification of $P$ such that the functions in $Q^{*}$ are precisely the restrictions of continuous functions on $K$. Let IT be the smallest $\sigma$-algebra of subsets of $P$ such that each element of $Q^{*}$ is measurable. Notice that the zero set of any function in $a^{*}$ is the intersection of a zero set in $K$ with $P$. It shows that

$$
\mathfrak{N}=P \cap[\operatorname{Baire}(K)]=E\{P \cap X \mid X \in \operatorname{Baire}(K)\} .
$$


Clearly all functions in $Q$ are measurable with respect to $\mathscr{T}$ ( $Q$ is a sublattice of $F(P)$ ). We shall show that none of the conditions $\mathrm{B}-\mathrm{F}$ is satisfied with $\mathfrak{T}$ and $a$ as defined above. The crucial fact is that: there is no $X$ in $\mathscr{T}$ with $Z_{1} \subset X \subset P-Z_{2}$ (whence $Z_{1} \notin \mathfrak{N}$ ). This fact follows immediately from Lemma 3 . Indeed, if there were such an $X$, then there would exist a countable subset $\mathcal{F}$ of $Q$ which would distinguish $M$. But this would contradict the fact that all elements of $\mathcal{F}$ are constant on the complement of some member $U$ of $น$. Indeed $P-U$ meets both $Z_{1}$ and $Z_{2}$.

Since $Z_{1} \notin \mathbb{T}$, $\mathfrak{T}$ does not contain Baire $(P)$, and hence condition B is not fulfilled. Next, $P$ is not Baire embedded in $K$ because $Z_{1} \notin \Re$; hence condition $\mathrm{C}$ is not fulfilled. To prove that condition $\mathrm{D}$ is not satisfied, consider a continuous function $f$ on $P$ which is 0 on $Z_{1}$ and 1 on $Z_{2}$. By the "crucial" fact the function $f$ is not measurable, and hence, by Lemma $3, f$ is not a Baire function with respect to $a$. Finally, each of conditions $\mathrm{E}$ and $\mathrm{F}$ implies condition $\mathrm{D}$, and hence neither $E$ nor $F$ is satisfied by $Q$. (Recall that $Q$ is a lattice.)

2.2.2. Evidently, condition $\mathrm{B}$ implies condition $\mathrm{C}$ as well as condition $\mathrm{D}$.

2.2.3. We shall prove that condition $A$ implies condition $B$. Assuming condition $\mathrm{A}$, it is enough to show that every zero set $Z$ belongs to शT. If $Z$ is Lindelö then $Z \in \Re$ by the argument in 2.1. If $Z$ is not Lindelöf then $P-Z$ is a countable union of Lindelöf zero sets, and hence $P-Z \in \mathscr{T}$.

2.2.4. ${ }^{2}$ It remains to show that condition $A$ implies conditions $\mathrm{E}$ and $\mathrm{F}$. Assume condition A. Let $Q$ be an algebra of bounded functions on $P$ which projectively generates the topology of $P$. Denote by $B$ the smallest lattice algebra containing $Q$. Since the smallest uniformly closed algebra $\mathfrak{e}$ containing $a$ is a lattice, we have $a \subset B$ $\mathcal{C}$, and hence it is obvious that it is enough to prove that every nonnegative continuous function is the pointwise limit of an increasing sequence of nonnegative functions in $B$.

If $U$ is an open Lindelöf subspace of $P$, then there exists an increasing sequence $\left\{g_{n}\right\}$ of nonnegative functions in $B$, which converges pointwise to the characteristic function of $U$.

Proof. Since $U$ is Lindelöf, there exists a sequence $\left\{h_{n}\right\}$ of nonnegative functions in $B$ such that $h_{n}[P-U] \subset(0)$, and if $x \in U$ then $h_{n} x>0$ for some $n$. Put

$$
g_{n}=\min \left(1, n\left(h_{1}+\cdots+h_{n}\right)\right) .
$$

${ }^{2}$ I am pleased to thank A. Hager for pointing out an error in the earlier version of 2.2.4. 
Now let $f$ be a nonnegative continuous function on $P$. First assume that $P$ is Lindelö; then all cozero sets are Lindelöf, and we can proceed as follows. Given an $\epsilon>0$, there exists a function $h$ which is the sum of a series of $\epsilon$ multiples of the characteristic functions of cozero sets in $P$, such that $h \leqq f$, and $\|f-h\|<\epsilon$; say $h=\epsilon \cdot \sum h_{n}$. By the italicized statement above, each $h_{n}$ is the limit of an increasing sequence $\left\{g_{n m}\right\}$ of nonnegative functions in B. Now, for $\epsilon=1 / k$, $k=1,2, \cdots$, let $f_{i}$ be the maximum of all $g_{n m}, n \leqq i, m \leqq i, g_{n m}$ corresponding to $\epsilon=1 / k, k \leqq i$. Evidently $\left\{f_{i}\right\}$ is an increasing sequence of nonnegative functions in $B$, and $\left\{f_{n}\right\}$ converges pointwise to $f$. This shows that conditions $\mathrm{E}$ and $\mathrm{F}$ hold if $P$ is Lindelöf. It should be remarked that this is Theorem 1 of A. Hager in [5].

Finally assume that condition A holds. This case is reduced to the previous one as follows. By Lemma 4 there is a real number $r$ such that $U=E\{x \mid f x \neq r\}$ is Lindelöf. We may assume that $r=0$. Apply the previous case of the restriction of $f$ to $U$, and to the restriction of $B$ to $U$. We get a sequence $\left\{h_{n}\right\}$ in $B$ such that $\left\{h_{n} \mid U\right\}$ is an increasing sequence of nonnegative functions, which converges to $f \mid U$. By the italicized statement above we can choose $g_{n}$ in B. Put $f_{n}=g_{n} \cdot h_{n}$. This concludes the proof.

2.2.5. REMARK. In $\mathrm{D}$ and $\mathrm{F}$ the assumption that the functions in $a$ are bounded may be deleted. Indeed, if $Q$ is a lattice then the proof in 2.2.4 works. If $a$ is not a lattice, then we can take a lattice $\$$ of bounded functions in $u Q \cap C(P)$, and apply the "bounded" case to get that $u^{2} Q \supset C(P)$. I think that one can prove that $u Q \supset C(P)$.

2.3. Proof of Theorem 3. Assume that $P$ is an analytic space, and $Q$ satisfies the assumption in Theorem 3. Let $Q$ be the topological space projectively generated by $Q$. The identity mapping $f$ of $P$ onto $Q$ is continuous, and since $P$ is analytic, Baire $(P)=\operatorname{Baire}(Q)$ by Theorem 2 in [2], or Theorem in [3]. Thus the Baire functions on $P$ coincide with the Baire functions on $Q$. Since $P$ is analytic, $P$ is Lindelöf, and hence $Q$ is Lindelöf. By Theorem 2 the algebra $a$ is dense in the Baire function on $Q$ in the sequential topology. This completes the proof because every continuous function on $P$ is a Baire function on $Q$.

REMARK. It may happen that if $\left\{f_{n}\right\}$ in $a$ converges to a continuous function $f$ on $P$, then $f \in Q$. For example, take a Baire set $Z$ of the order 2 in an analytic space $Q$, and let $P$ be the space projectively generated by the characteristic function $g$ of $Z$, and all continuous functions on $Q$. Put $Q=\mathfrak{e}(Q)$.

2.4. Proof of Theorem 4. Assume that a space $P$ is almost Lindelöf but not Lindelöf. We shall show that $P$ is not Baire-minimal. 
Let $\mathrm{Z}$ be the collection of all zero sets in $P$ which are not Lindelöf. Clearly $\mathrm{Z}$ is a maximal filter of zero sets, and $\mathrm{Z}$ has the countable intersection property. Since $P$ is not Lindelö, it is immediate that $\cap \mathrm{Z}=\varnothing$. Choose a point $a$ in $P$, and consider the set of all continuous functions $f$ on $P$ such that $(f x)=f[Z]$ for some $Z$ in $Z$. It is easy to check that $Q$ is a uniformly closed algebra which separates the points of $P$, and if $g$ is a continuous function on $P$ such that the zero set of $g$ belongs to $Z$ and $g x \neq 0$, then (Lemma 3 ) $g$ is not a Baire function with respect to $Q$

2.5. Proof of Theorem 5. We shall need the following well-known result (I do not know of any reference).

2.5.1. Proposition. Let $g$ be a continuous mapping of a space $P$ onto a space $Q$. If $P$ has an open base of cardinal $\chi$, then there exists a collection $\mathcal{F}$ of continuous functions on $Q$, which distinguishes the points of $Q$, such that the cardinal of $\mathcal{F}$ is at most $\chi$.

PRoof. Let $\mathcal{U}$ be an infinite open base for $P$, and for each pair $U, V \in \mathcal{U}$ choose a continuous function $f_{U V}$ on $Q$ which is, if possible 0 on $U$ and 1 on $V$. The family $\left\{f_{U V}\right\}$ distinguishes the points of $Q$.

2.5.2. Assume that $g$ is a one-to-one continuous mapping of a Blackwell space onto a space $Q$. By 2.5.1 there exists a one-to-one continuous mapping $h$ of $Q$ onto a separable metrizable space $M$. The composite $f=h \circ g$ is a one-to-one Baire measurable mapping, and Baire $(M)$ is countably generated. Hence $f$ is an isomorphism, and it follows that $g$ is an isomorphism.

2.5.3. Assume that $\langle P, B\rangle$ is a countably generated measurable space which is not a Blackwell space, and take a countably generated proper subalgebra $B_{1}$ of $B$ which distinguishes points. Let $\left\{B_{n}\right\}$ be a generating sequence for $B$ such that $\left\{B_{2 n}\right\}$ generates $B_{1}$. For each $n$ let $i_{n}$ be the characteristic function of $B_{n}$. Let $Q$ be the product of $\{2 \mid n \in N\}$, where 2 is the discrete space consisting of 0 and 1 , and let $Q_{1}$ be the product of $\{2 \mid n / 2 \in N\}$. Denote by $\pi$ the projection of $Q$ onto $Q_{1}$. Then $\langle P, B\rangle$ is measurably embedded into $Q$ by $i x=\left\{i_{n} x\right\}$, $\left\langle P, Q_{1}\right\rangle$ is measurably embedded by $j x=\left\{i_{2 n} x\right\}$, and clearly $j=\pi \circ i$. Thus the topology induced by $i$ is not Baire-minimal.

\section{REFERENCES}

1. E. Cech, Topological spaces, 2nd ed., Publ. House Czech. Acad. Sci., Prague, 1965; English transl. of 1st ed., Wiley, New York, 1966. MR 35 \#2254.

2. Z. Frolik, A measurable map with analytic domain and metrizable range is quotient, Bull. Amer. Math. Soc. 76 (1970), 1112-1117.

3. - A survey of descriptive theory of sets and spaces, Czechoslovak Math. J. 20 (1970), 406-467. 
4. Z. Frolik, A contribution to the descriptive theory of sets and spaces, Proc. Sympos. General Topology and its Relations to Modern Analysis and Algebra (Prague, 1961), Academic Press, New York; Publ. House Czech. Acad. Sci., Prague, 1962, pp. 157173. MR $26 \# 3002$.

5. A. W. Hager, Approximation of real continuous functions on Lindelöf spaces, Proc. Amer. Math. Soc. 22 (1969), 156-163. MR 39 \#6062.

6. A. W. Hager and D. G. Johnson, $A$ note on certain subalgebras of $C(X)$, Canad. J. Math. 20 (1968), 389-393. MR 36 \#5697.

7. E. Hewitt, Certain generalizations of the Weierstrass approximation theorem, Duke Math. J. 14(1947), 410-427. MR 9, 95.

Mathematics Institute of the Czechoslovak Academy of Sciences, Prague, Czechoslovakia

State University of New York, Buffalo, New York 14266. 